\title{
Social Media's Impact on Creative Thought
}

\author{
Lauren Robinson \\ School of Psychology \\ University of East London \\ Janine Streuli \\ Faculty of Education \\ Royal Academy of Dance
}

\author{
Antonio Rei Fidalgo \\ School of Psychology \\ University of East London
}

\begin{abstract}
Social media is a powerful force in our modern-day world with several published studies that suggest negative consequences due to its extended use. This experiment scrutinizes whether social media affects creativity by investigating how short-term exposure to social media impacts an individual's capacity for creative thought, as measured by the Guilford Alternate Uses Test and the Remote Associations Test. Results suggest no statistically significant difference on the Guilford Alternate Uses Test while the Remote Associations Test performance appears to be impacted. Limitations of the experimental design, such as questionnaires used and further areas of study topics are suggested.
\end{abstract}

Keywords: social media; creative thought;

\section{INTRODUCTION}

The incredibly rapid technological advancements and development of mobile phones has led to devices that can be used as a phone, computer, camera, radio, television, calculator, blogging tool, diary, bank, or a book [1]. Recent research suggest that mobile phone usage can be associated with a range of negative mental health issues some of which include: the breakdown of interpersonal relationships [2]; interrupted sleep [3]; a desire to use immediately upon waking, a behaviour akin to that of an addiction [2]; anxiety when separated from our device [2]; accidents due to lack of attention when walking and driving [2]; and an inability to relax due to a perpetually heightened state of mental activity [2].

In a similar manner, studies that focus on social media usage have often associated the impact of its use as having a negative effect on adolescents' mental health [4] with reports linking increased use with higher levels of anxiety [5]; the likelihood of increased depression scores [6]; and negative impact on GPA (an indicator of academic attainment in the USA), which was also seen to be more pronounced in females than males [7].

One notion that seems to have gone un-investigated is the effect that mobile phone use might be having on our capacity to think creatively. The field of creativity can largely be credited to the work of Guilford and Torrance [8] with the
Torrance Test of Creative Thinking [8] still being the most widely used assessment of creative talents [9]. While the "Remote Associations Test" is used to measure convergent thinking [10].

Research conducted by Kim [11] suggests that performance in the Torrence Test declined in US school populations from 1990-2008. This was particularly evident below grade three, with adolescents experiencing a leveling-off period which was thought to be linked to the need to conform to school expectations and social pressures. Previous research by Runco [12] also suggests that age plays a factor in general creativity levels. Young children appear to experience a decline in general creativity around the age of 5 or 6 [13] followed by a subsequent increase of general creativity until puberty when there is another decline [13].

This study aims to scrutinize whether we can observe any short-term effect on creativity test scores after social media browsing on a mobile phone device.

\section{METHODS}

Participants: 39 female students from a private all-girls school in Central London aged between 16-18 (M=16.69, $\mathrm{SD}=0.73$ ) took part in this experiment. The study was approved by the Ethics Committee of the University of East London and run in accordance with the Declaration of Helsinki. All participants reported normal or corrected-tonormal vision and hearing.

\section{Materials}

Kaufman Domains of Creativity Scale (K-DOCS): a self-report list of creative behaviours originally derived from the Creativity Domain Questionnaire [14]. Individuals are asked to read a series of 50 statements and rate themselves on a 5-point Likert Scale, with regard to how creative they perceived themselves to be in relation to others of a similar age and life experience. Each area was given a total score out of 25 to determine the individual's self-report measure with the highest possible score being 250 . 
Guildford Alternate Uses (GAU) Test: in this test participants suggest alternative uses to 2 common objects (e.g.: brick) with a time limit of 4 minutes for each object. Upon completion the GAU responses were processed through a series of parameters to determine scores (determining the total number of uses generated; the novelty of the use; and the type of uses). The novelty of the use was determined by the number of other participants that had also suggested it, if two or more also offered the same suggestion, it was not considered novel [15].

Remote Associations Test (RAT): In this test there were ten groups of words in total and participants were told, after 15 seconds had elapsed, to move onto the next set. The word sets gradually got harder in terms of finding associative links that made sense for all of the words in the set. Participants can receive a maximum score of 10 for each set. Often, the link for the words is not immediately obvious and so individuals are required to be 'creative' in their thought [13].

Procedure: participants were given the K-DOCS, GAU and RAT to complete beforehand. Then were divided into either Control or Social Media conditions. For the Control condition they were asked to read a section of text from 'Sociology Today' magazine for 10 minutes; while for the Social Media condition they were instructed to use their mobile phone to browse on social media for 10 minutes. After this manipulation, participants were asked to repeat the GAU and RAT.

\section{RESUlts}

Results showed that pupils estimated their total phone use on one weekday and one weekend day to be between 40 minutes to 20 hours $(\mathrm{M}=7.5$ hours, $\mathrm{SD}=4.08)$. There was no statistically significant different between groups $(p>0.05)$.

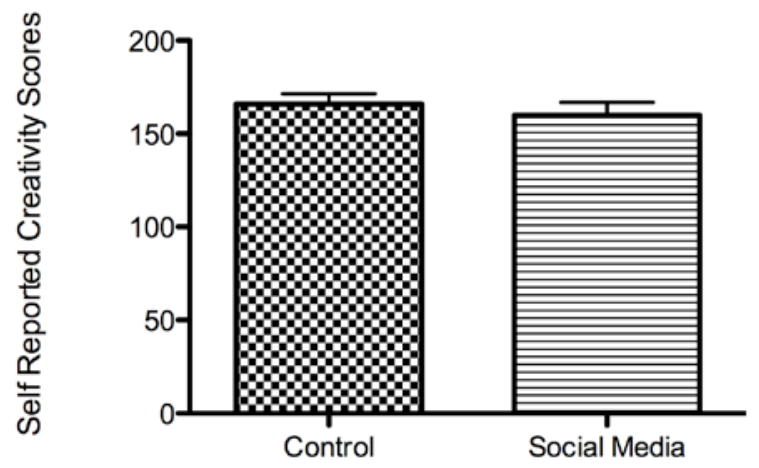

Fig 1: The overall self-reported creativity scores are similar between both groups.
Their self-reported creativity levels ranged from 109 to 234 $(\mathrm{M}=162.62, \mathrm{SD}=28.41)$ attainable (see Figure 1). There was no statistically significant different between groups $(p>0.05)$.

Initial scores on the GAU test ranged from 6 to $22(\mathrm{M}=13.081$, $\mathrm{SD}=3.402)$. The higher the score, the higher the creativity rating (see Figure 2). Results suggest no statistically significant differences between the pre and post GAU test results $F(1,35)=2.375, p=0.132$. There was also no significant difference between these results when participants were in different conditions $F(1,35)=0.08, p=0.779$.

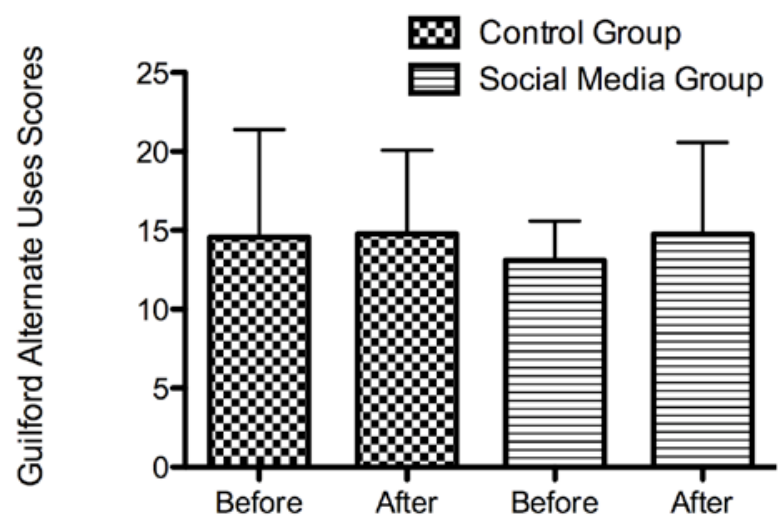

Fig 2: the pre- and post-intervention scores on the Guilford Alternate Uses were not influenced by the conditions.

The RAT test scores ranged from 0 to $5(\mathrm{M}=2.027$, $\mathrm{SD}=1.301$ ) with the highest attainable score being 10 (see Figure 3 ). There was a significant difference between the pre and post RAT results $F(1,35)=16.812, p=0.001$. There was also a significant difference between the results based on the groups participants were placed in $F(1,35)=7.596, p=0.009$. Despite these results, the interaction between test results in relation to the condition candidates were placed in was not significant $F(1,35)=2.559, p=0.119$.

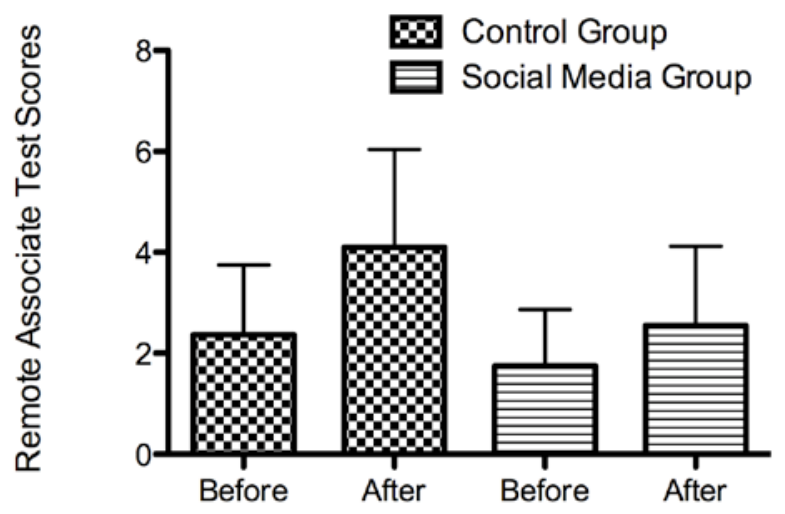

Fig 3: the pre- and post-intervention scores on the Remote Associate Test are influenced by the conditions. 


\section{DISCUSSION}

The results suggest that the short-term exposure that participants had to social media on their device had no overall impact on their cognitive capacity to think creatively. This, in conjunction with the results from the GAU and RAT rests would appear to suggest that no direct impact on creative thought can be linked to short-term social media exposure which poses some interesting considerations.

Participants in this study provided an estimation of their mobile phone usage. Weekday use was perceived to be higher than weekends, despite phones not being allowed during school hours. This is actually contrary to findings in other research where the weekend period tends to see more social media use [16]. Literature on self-reported mobile phone use has also suggested that individuals are often poor at accurately estimating how much they use their phones $[16,17]$. A study by Lee et al. [18] reports that college students can underestimate smartphone use by as much as $40 \%$. However, there are now apps that can monitor smartphone usage accurately $[16,18]$.

Despite our results suggesting no direct link between creativity and social media use, others propose an indirect link [19]. Children are spending much less time outdoors maybe due to an increased exposure to screens and social media [19]. This lack of exposure to external stimulation as well as the tribulations and interactions that arise from playing outside with others may results in a generation of children who have not learnt to judge risk correctly and not develop adequate resilience skills [19]. In a similar manner reduced physical activity is also known to have serious health implications [20].

If creativity can be influenced by those that we choose to interact with, then increasing the number of people who have access to social media would be beneficial and enhance creativity [21]. Specially, exposure to different points-of-view in the form of opinions, audio-visual materials, news-stories, stories and other emotionally salient material may be useful as it allows individuals to engage with content they might not normally chose to be exposed to, and in so doing, broaden their horizons [22].

Social media may be perceived negatively due to reports linking its use with negative impact on interpersonal relationships [2], health [3] and, mental health [4, 5, 6]. In addition to this, we often see screen time suggestions by professional bodies [24] and requests for guidelines to be issued by the government to manage screen time [24]. Therefore, there is a high need for research findings on the different ways in which social media can impact in an individual's life. Our findings do not suggest that a direct link between creativity levels and short-term social-media browsing.
While it may be the case that passive social media use negativity impacts a range of psychological functions [2, 4] among which may be creativity $[5,8]$, creativity is comprised of four cognitive stages: preparation, incubation, illumination and verification $[8,12]$. The internet and social media can assist in preparation by making information easily accessible [1]. While creative discovery often spurs from a conscious break of thought $[5,12]$, prolonged use of social media may negativity impact incubation as it does with other psychological functions [2, 4]. In a similar manner, social media may help with illumination $[8,12]$ by allowing the recording of the idea, which can then be followed by verification $[8,12]$ by facilitating an almost instant feedback from others [22].

Despite the fact that the Kaufman Domains of Creativity Scale is considered a viable self-report measure, it has been previously reported that an individual's view of their creativity in relation to expert opinion is often mismatched [20] with people tending to hold overly favorable views of their abilities in many social and intellectual domains [20]. In a similar manner, the scoring of the Guildford Alternate Uses test has also received criticism as a person who is more fluent in their response is also more likely to score highly in the novelty category [25]. The Kaufman Domains of Creativity Scale and Guildford Alternate Uses test have been shown to be directly related to one another [25]. In our study, we also applied strict time restrictions on participants when completing the tests. This is particularly relevant as previous research [26] suggests that time pressure was a negative influence on creativity.

As studies suggest that creativity levels are declining [11] it may be worthwhile for researchers to consider what factors may be contributing to this. This may involve long-term studies both on the use of social media, smartphone usage and additional measures of creativity as well as an assessment of social media's impact on time available/used for outdoor play, face-to-face social interaction and other free play. Another interesting point to consider for further research is the discrepancy that exists between creativity in a school setting and outside of academic settings. It is widely reported that there is a 'creativity gap' whereby undergraduate students produce less creative work in an academic setting than work that they produce while in a work setting [13].

In conclusion, the findings from our study suggest that, although no significant short-term effects can be observed on creativity levels (as measured by the Guildford Alternate Uses test and the Remote Associations Test) in relation to social media use, there are still many reasons why further research into this area would be beneficial to the wider public. The lack of long-term data in relation to social media use is a key issue in the existing research. 


\section{ACKNOWLEDGMENT}

We would like to thank our participants for taking part in the research project. In a similar manner we are grateful to the school management team for supporting this project.

\section{REFERENCES}

[1] A. Lepp, J. Barkley, and A. Karpinski, "The relationship between cell phone use, academic performance, anxiety, and Satisfaction with Life in college students," Comput Human Behav, vol. 31, pp. 343-350, 2014

[2] C. Yildirim, and A.P. Correia, "Exploring the dimensions of nomophobia: development and validation of a self-reported questionnaire," Comput Human Behav, vol. 49, pp. 130-137, 2015.

[3] L. Hale, and S. Guan, "Screen time and sleep among school-aged children and adolescents: a systematic literature review," Sleep Med Rev, vol. 21, pp. 50-58, 2015.

[4] E. Firth, "Social media and mental health: a review of the evidence," Retrieved March 2, 2018, from https://epi.org.uk/wpcontent/uploads/2018/01/Social-Media_Mental-Health_EPI-Report.pdf, 2017

[5] H. Sampasa-Kanyinga, and R.F. Lewis, "Frequent Use of Social Networking Sites Is Associated with Poor Psychological Functioning Among Children and Adolescents," Cyberpsychol Behav Soc Netw, vol. 18 , pp. 380-385, 2015

[6] E.M. Seabrook, M.L. Kern, and N.S. Rickard, "Social Networking Sites, Depression, and Anxiety: A Systematic Review," JMIR Ment Health vol. 3(4):e50., 2016.

[7] L. Dong, P. Kirschner, and C.A. Karpinski "A meta-analysis of the relationship of academic performance and Social Network Site use among adolescents and young adults," Comput Human Behav, vol. 77, pp. 148-157, 2017.

[8] R.J. Sternberg, “The Nature of Creativity," Creat Res J, vol. 18, pp. $87-$ 98, 2006.

[9] T. Lubart, and Z. Zenasni, "Creative Potential and its Measurement," International Journal for Talent Development and Creativity, 1, 41-51, 2013.

[10] M. E. Backman, and B.W. Tuckman. "Review of Remote Associates Test," J Educ Meas, vol 9, pp 161-162, 1972.

[11] K.H. Kim (2011) "The Creativity Crisis: The Decrease in Creative Thinking Scores on the Torrance Tests of Creative Thinking," Creat Res J, vol. 23, pp. 285-295, 2011.
[12] M.A. Runco, “Creativity,” Annu Rev Psychol, vol. 55, pp. 657-87, 2004.

[13] M.A. Runco, S. Acar, and N. Cayirda, "A closer look at the creativity gap and why students are less creative at school than outside of school," Think Skills Creat., vol. 24, pp. 242-249, 2016

[14] J.C. Kaufman, "Counting the Muses: Development of the Kaufman Domains of Creativity Scale (K-DOCS),'. Psychol Aesthet Creat Arts, vol. 6, pp. 298-308, 2012

[15] M. Oppezzo, and D.L. Schwartz, "Give Your Ideas Some Legs: The Positive Effect of Walking on Creative Thinking. J Exp Psychol Learn Mem Cogn, vol. 40, pp. 1142-1152, 2014.

[16] J. Boase, and R. Ling "Measuring Mobile Phone Use: Self - Report Versus Log Data," J Comput Mediat Commun, vol. 18, pp. 508-519, 2013.

[17] M.V. Abeele, K. Beullens, and K. Roe, "Measuring mobile phone use: Gender, age and real usage level in relation to the accuracy and validity of self-reported mobile phone use," Mobile Media \& Commun, vol. 1, pp. 213-236, 2013.

[18] H. Lee, H. Ahn, T.G. Nguyen, S.-W. Choi and, J.D. Kim, “Comparing the Self-Report and Measured Smartphone Usage of College Students: A Pilot Study," Psychiatry Investig, vol. 14, pp. 198-204, 2017.

[19] M. Brussoni, L.L. Olsen, I. Pike, and D.A. Sleet, "Risky Play and Children's Safety: Balancing Priorities for Optimal Child Development," Int J Environ Res Public Health, vol. 9, pp. 3134-3148, 2012.

[20] K. Sahoo, B. Sahoo, A.K. Choudhury, N.Y. Sofi, R. Kumar, and A.S Bhadoria, "Childhood obesity: causes and consequences", J Family Med Prim Care, vol. 4, pp. 187-192, 2015.

[21] J.H. Holland, "Emergence: From Chaos to Order," Oxford University Press, 1998

[22] A.S. Kumpel, V. Karnowski, and T. Keyling, "News Sharing in Social Media: A Review of Current Research on News Sharing Users, Content, and Networks," Social Media + Society, vol. 1, pp. 2-14, 2015.

[23] J. Kruger, and D. Dunning, "Unskilled and unaware of it: How difficulties in recognizing one's own incompetence lead to inflated selfassessments," J Pers Soc Psychol, vol. 77, pp. 1121-1134, 1999.

[24] American Academy of Pediatrics, "Media and Young Minds," Pediatrics, vol. 138, e20162591, DOI: 10.1542/peds.2016-2591, 2016.

[25] E.P. Torrance, "Predicting the creativity of elementary school children (1958-1980) - and the teacher who made a "difference", Gifted Child Q, vol. 25 , pp. $55-62,1981$.

[26] T.M Amabile, J.S. Mueller, W.B. Simpson, L.Fleming, and C. N Hadley. "The Influence of Time Pressure on Creative Thinking in Organizations." Paper presented at the Academy of Management Annual Meeting, Seattle, WA, August 01-06, 2003. 